\title{
Noxa determines localization and stability of MCL-1 and consequently ABT-737 sensitivity in small cell lung cancer
}

\author{
W Nakajima ${ }^{1}$, MA Hicks ${ }^{1}$, N Tanaka ${ }^{2}$, GW Krystal ${ }^{3}$ and H Harada ${ }^{\star, 1}$
}

The sensitivity to ABT-737, a prototype BH3 mimetic drug, varies in a broad range in small cell lung cancer (SCLC) cells. We have previously shown that the expression of Noxa, a BH3-only pro-apoptotic BCL-2 family protein, is the critical determinant of ABT-737 sensitivity. We show here that Noxa regulates the localization and stability of MCL-1, an anti-apoptotic member, which results in modulating ABT-737 sensitivity. Mutations in Noxa within the BH3 domain, the carboxyl terminus mitochondrial targeting domain, or of ubiquitinated lysines not only change the localization and stability of Noxa itself but also affect the mitochondrial localization and phosphorylation/ubiquitination status of MCL-1 and consequently modulate sensitivity to ABT-737. Results of studies utilizing these mutant proteins indicate that Noxa recruits MCL-1 from the cytosol to the mitochondria. Translocation of MCL-1 initiates its phosphorylation and subsequent ubiquitination, which triggers proteasomemediated degradation. The precise regulatory mechanisms of Noxa/MCL-1 expression and stability could provide alternative targets to modulate apoptosis induced by $\mathrm{BH} 3$ mimetic drugs or other chemotherapeutic reagents.

Cell Death and Disease (2014) 5, e1052; doi:10.1038/cddis.2014.6; published online 13 February 2014

Subject Category: Cancer

Small cell lung cancer (SCLC) accounts for approximately $15 \%$ of lung cancers diagnosed in the US and causes a fatality rate of $>90 \% .^{1}$ Paradoxically, SCLC was one of the first epithelial malignancies in which combination chemotherapy could increase median survival, with a variety of multi-drug combinations producing response rates of $>80 \%$, albeit with the vast majority suffering recurrence. ${ }^{2}$ Over the past 25 years, progress has been made in limited-stage SCLC, where the combination of concurrent radiation and chemotherapy has resulted in a long-term survival rate of $20-25 \%$. However, progress has been slow in extensive SCLC ( $70 \%$ of all cases), with long-term survival rates of approximately $3 \% .{ }^{3-5}$

Tumor cell death induced by chemotherapy or lack of appropriate cellular survival signals is mediated by the intrinsic apoptotic pathway. The integrity of the outer mitochondrial membrane is regulated by the balance of antiand pro-apoptotic members of the B-cell lymphoma (BCL)-2 protein family. ${ }^{6,7}$ The BCL-2 family is subdivided into three main groups based on regions of BCL-2 homology $(\mathrm{BH})$ and function: multidomain anti-apoptotic (BCL-2, myeloid cell leukemia sequence $\left.1(\mathrm{MCL}-1), \mathrm{BCL}-\mathrm{X}_{\mathrm{L}}\right)$, multidomain proapoptotic (BAX, BAK), and BH3-only pro-apoptotic (e.g., BCL-2 interacting mediator of cell death (BIM), PUMA, Noxa). The $\mathrm{BH} 3-$ only proteins clearly act upstream of BAX and BAK, because they cannot induce apoptosis in cells lacking both
BAX and BAK. BH3-only proteins cause cytochrome $c$ release by activating $B A X$ and/or BAK, and the anti-apoptotic BCL-2 family of proteins prevents this process. We and the others have demonstrated that the anti-apoptotic member BCL-2, as well as BCL- $X_{L}$ and MCL-1, is overexpressed in SCLC. ${ }^{8,9}$ However, until recently, the precise role of these proteins in SCLC biology and therapeutic resistance was poorly understood. The breakthrough came with the development of $\mathrm{BH} 3$ mimetic antagonists that block the function of pro-survival BCL-2 family members. ABT-737, the prototype of this new drug class, binds to and blocks BCL-2 and BCL- $\mathrm{X}_{\mathrm{L}}$, but not MCL-1, function. ${ }^{10} \mathrm{BH} 3$ mimetic drugs represent one of the most potentially exciting breakthroughs in cancer therapy. Not only do they appear effective in selected malignancies that are highly dependent on the function of the anti-apoptotic BCL-2 family proteins but also act synergistically with chemotherapeutic agents and radiation against a wide variety of malignancies. ${ }^{10-12}$ SCLC is the only non-hematological malignancy against which ABT-737 and its orally available derivative ABT-263 (currently undergoing clinical trials) are effective as a single agent, albeit with a broad range of sensitivities among established cell lines. ${ }^{10,13,14}$ SCLC's response rates to ABT-263 in early clinical studies have also been quite variable, ranging from minimal tumor shrinkage to progressive disease. ${ }^{14}$ It was demonstrated that one factor

\footnotetext{
${ }^{1}$ Department of Oral and Craniofacial Molecular Biology, School of Dentistry, Massey Cancer Center, Virginia Commonwealth University, Richmond, VA, USA; ${ }^{2}$ Department of Molecular Oncology, Institute of Gerontology, Nippon Medical School, Kawasaki, Japan and ${ }^{3}$ Department of Internal Medicine, Virginia Commonwealth University, McGuire Veterans Affairs Medical Center, Richmond, VA, USA

${ }^{*}$ Corresponding author: H Harada, Department of Oral and Craniofacial Molecular Biology, School of Dentistry, Massey Cancer Center, Virginia Commonwealth University, 401 College Street, Richmond, VA 23298, USA. Tel: +1 804628 0064; Fax: +1 804827 1276; E-mail: hharada@ vcu.edu

Keywords: ABT-737; lung cancer; MCL-1; Noxa; protein stability

Abbreviations: SCLC, small cell lung cancer; MCL-1, myeloid cell leukemia sequence 1; BCL, B-cell lymphoma; BH, BCL-2 homology; BIM, BCL-2 interacting mediator of cell death; shRNA, short-hairpin RNA

Received 05.9.13; revised 16.12.13; accepted 02.1.14; Edited by G Ciliberto
} 
mediating in vitro resistance to ABT-737 was the level of MCL-1 expression. ${ }^{11,15,16}$ However, we previously noted that MCL-1 expression was similar in a panel of SCLC cell lines whose sensitivity to ABT-737 varied over 2 logs. We also noted that Noxa (a pro-apoptotic BH3-only protein that specifically binds MCL-1) expression directly correlated with sensitivity to ABT-737. ${ }^{17}$ Here, we further examined the molecular mechanisms of ABT-737 sensitivity regulated by the NOXA/MCL-1 axis. We show that Noxa regulates MCL-1 localization and stability by: (1) BH3-mediated protein:protein interaction; (2) mitochondrial targeting of the protein complex; and (3) ubiquitination of lysine residues in Noxa leading to phosphorylation/ubiquitination of MCL-1. ABT-737's sensitivity of SCLC is modulated as a consequence of these processes that regulate $\mathrm{MCL}-1$ expression.

\section{Results}

The level of Noxa expression in SCLC cells correlates with the sensitivity to ABT-737. To identify factors that were responsible for variable sensitivity of SCLC to ABT-737, we used a representative panel of SCLC cell lines with levels of sensitivity that ranged over $>2$ logs $\left(\mathrm{IC}_{50}\right.$ : $0.03 \sim>10 \mu \mathrm{M})$. Expression of the BCL-2 family members, particularly MCL-1, Noxa, and BIM, which has been implicated in determining sensitivity to ABT-737, was assessed in this panel of cell lines (Figures 1a-d). MCL-1 levels were relatively constant in all cell lines. Among the eight cell lines we examined, four groups could be separated based on Noxa and BIM expression: (1) Noxa-high, BIM-low (H1184 and H2171); (2) Noxa-high, BIM-high (H69 and H2141); (3) Noxa-low, BIM-high (H128 and H526); and (4) Noxa-low, BIM-low (H82 and H209). In group 4, both Noxa and Bim mRNAs and their protein expression were undetectable in $\mathrm{H} 209$, and both BCL-2 and BCL- $\mathrm{X}_{\mathrm{L}}$ expression was significantly lower in $\mathrm{H} 82$ than those in other cell lines. The extent of apoptosis with $100 \mathrm{nM} \mathrm{ABT-737}$ in each cell line was confirmed by PARP cleavages, which was consistent with the $\mathrm{IC}_{50}$ values (Figure 1e).

In order to examine the significance of Noxa and BIM for sensitivity to ABT-737, we introduced short-hairpin RNAs (shRNAs) for Noxa and Bim in H2171, H69, and H526. In the cases of Noxa-high cells ( $\mathrm{H} 2171$ and $\mathrm{H} 69)$, downregulation of Noxa strongly inhibited apoptosis induced by ABT-737 judged by PARP cleavage (Figures $2 a$ and $b$ and Supplementary Figure S1). Downregulation of BIM in $\mathrm{H} 69$ cells (BIM-high) did not significantly affect the extent of ABT-737-induced apoptosis (Figure $2 b$ ), suggesting BIM is dispensable in this cell line. In contrast, downregulation of BIM strongly inhibited apoptosis induced by ABT-737 in H526 cells, while downregulation of Noxa only caused partial inhibition (Noxa-low, BIM-high) (Figure 2c). These results suggest that Noxa is primarily responsible for $\mathrm{ABT}-737$ sensitivity and that BIM contributes to the sensitivity if Noxa expression is low.

As H209 cells showed undetectable levels of Noxa and BIM and were resistant to ABT-737, we introduced Noxa or BIM cDNAs into $\mathrm{H} 209$ cells to examine whether the expression of Noxa or BIM could restore ABT-737 sensitivity. When Noxa was expressed in $\mathrm{H} 209$ cells (Figure $3 \mathrm{a}$ ), apoptosis was increased over $16 \mathrm{~h}$ using only $10 \mathrm{nM}$ ABT-737 (Figure 3b).
It has been proposed that BAK is sequestered by MCL-1 and BCL- $X_{L}$ until displaced by BH3-only proteins. ${ }^{18}$ In $\mathrm{H} 209$ cells, Noxa bound MCL-1 regardless of ABT-737 treatment (Figure $3 \mathrm{c}$ ) and the binding between MCL-1 and BAK was decreased in the presence of Noxa (Figure 3d). The BAK$B C L-X_{L}$ interaction was decreased by $A B T-737$ binding to $B C L-X_{L}$ (Figure $3 d$ ), ${ }^{10}$ indicating that BAK was released from both $\mathrm{MCL}-1$ and $\mathrm{BCL}-\mathrm{X}_{\mathrm{L}}$ only when Noxa-expressing cells were treated with ABT-737. As a consequence, BAK was free from binding and became active (Figure 3e). ABT-737 is known to bind to BCL-2 as well as BCL- $X_{L}$. Therefore, BAX also became active in Noxa-expressing cells treated by ABT737 (Figure 3f). These results demonstrate that MCL-1 inactivation by Noxa together with inhibition of BCL-2 and $B C L-X_{L}$ by ABT-737 induces apoptosis. The exogenous expression of BIM in $\mathrm{H} 209$ cells also induced apoptosis with ABT-737 treatment (Figure $3 \mathrm{~g}$ ), presumably because BIM was capable of interacting with MCL-1. The result indicates that BIM has a significant role in the induction of apoptosis by ABT-737 when Noxa is absent, as shown in Figure 2c. When Noxa was transiently expressed in $\mathrm{H} 82$ cells, apoptosis was induced without ABT-737 treatment (Supplementary Figure S2), suggesting that MCL-1 inactivation by Noxa is enough to induce apoptosis if the expression of BCL-2 and BCL- $X_{L}$ is low (Figure 1a).

The expression of Noxa in $\mathrm{H} 209$ cells recruits MCL-1 to the mitochondria followed by the induction of its phosphorylation. It has been demonstrated that several domains and amino acids are responsible for the function of Noxa: (1) BH3 domain for the interaction with anti-apoptotic BCL-2 family proteins, such as MCL-1 and $A 1 ;{ }^{19}$ (2) lysine residues for ubiquitination; ${ }^{20}$ (3) C-terminus hydrophobic amino acids for the mitochondrial localization (mitochondria targeting domain (MTD)) ${ }^{21}$ In order to determine the role of these functional components in regulating $\mathrm{MCL}-1$ activity, we substituted corresponding amino acids to generate Noxa mutants 3E (BH3 domain), KR (ubiquitination sites), and 5A (MTD) (Figure 4a) and introduced them into H209 cells. The levels of Noxa and MCL-1 protein varied depending on the Noxa isoform expressed (Figure 4b), although the levels of exogenous Noxa and endogenous MCL-1 mRNA were similar in the cells (Figure 4c). The levels of Noxa-KR and Noxa-5A were higher and lower, respectively, than that of Noxa-wild type (wt), and the level of MCL-1 was significantly higher in the cells expressing Noxa-5A (Figure 4b), indicating post-transcriptional regulation.

We first examined the ability of each Noxa mutant to interact with MCL-1 by co-immunoprecipitation. The $\mathrm{BH} 3$ domain mutant Noxa-3E did not bind to MCL-1, but other mutants retained that ability (Figure $4 d$ ). As Noxa-MCL-1 interaction is critical for ABT-737 sensitivity (Figure 3), we next examined the induction of apoptosis with ABT-737 treatment in cells expressing each Noxa mutant. Consistent with the capability of Noxa-MCL-1 interaction, Noxa-3E could not induce apoptosis by ABT-737 treatment, and Noxa-KR and Noxa$5 \mathrm{~A}$ induced apoptosis more and less, respectively, compared with Noxa-wt, correlating with the levels of Noxa protein (Figure 4e). 


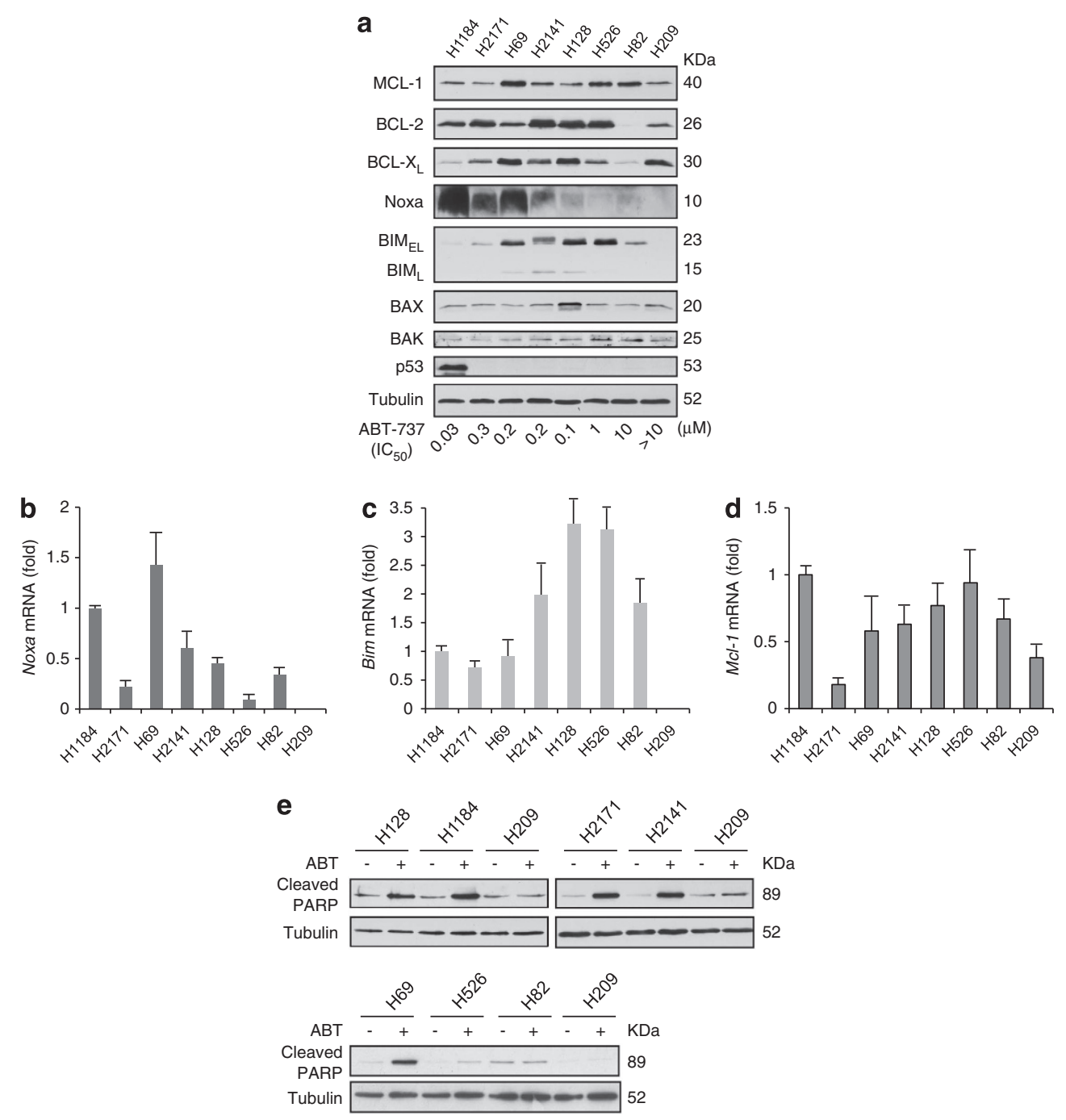

Figure 1 The expression levels of Noxa in SCLC cells correlate with sensitivity to ABT-737. (a) Expression of the BCL-2 family proteins and p53 in SCLC cell lines was determined by immunoblot analysis. The $\mathrm{IC}_{50}$ of ABT-737 in each cell line was determined by WST-1 assay at $72 \mathrm{~h}$ after treatment. (b-d) Noxa (b), Bim (c), and Mcl-1 (d) mRNA expression in SCLC cell lines was measured by quantitative real-time PCR (qPCR). Average values from triplicate samples are shown as representative of two independent experiments. (e) Apoptosis induced by ABT-737 $(100 \mathrm{nM})$ treatment for $24 \mathrm{~h}$ was detected by poly ADP-ribose polymerase (PARP) cleavage with immunoblot analysis

Noxa is known to be predominantly localized at the mitochondria. $^{21}$ Thus, we examined whether the mutation of Noxa affects its subcellular localization, as well as that of MCL-1. H209 cells were subcellularly fractionated into cytosol and heavy-membrane (HM; enriched for mitochondria) fractions. Although Noxa-wt, Noxa-3E, and Noxa-KR were predominantly localized at the mitochondria, Noxa-5A, in which all hydrophobic amino acids were substituted to alanines, was localized more in the cytosol (Figure 4f). Interestingly, the localization of $\mathrm{MCL}-1$ was also dramatically changed depending on the expression of Noxa-wt or its mutants. When Noxa-wt was expressed in $\mathrm{H} 209$ cells, most MCL-1 protein was clearly localized in the mitochondrial fraction. In contrast, the expression of Noxa-3E, which could not bind to MCL-1, did not affect MCL-1 localization compared with H209 cells without Noxa expression (Figure 4f, upper panel). Noxa-KR expression induced similar MCL-1 translocation as observed in Noxa-wt. Both Noxa-5A and MCL-1 were more prevalent in the cytosol. These results suggest that Noxa and MCL-1 co-localize when they physically interact and form a complex. We also noted several slower-migrating bands of MCL-1 on regular SDS-PAGE when Noxa-wt was expressed. Thus, we performed SDS-PAGE with Phos-Tag ${ }^{22}$ to examine the phosphorylation status of MCL-1 in Noxainfected cells. Slower-migrating bands indicative of phosphorylation of MCL-1 were detected only in the mitochondrial fraction when Noxa-wt or Noxa-KR was expressed in H209 cells (Figure 4f, lower panel). This result suggests that Noxa binds to and recruits MCL- 1 to the mitochondria and that MCL-1 is subsequently phosphorylated.

We next addressed whether Noxa could recruit MCL-1 from cytosol to the mitochondria in other cells. As the MCL-1 antibodies we tested were not suitable for immunofluorescence (they stained cells non-specifically; Supplementary Figure S3), we transfected myc-tagged MCL-1 (5Myc-MCL-1) into HeLa cells and detected the MCL-1 localization by 
a

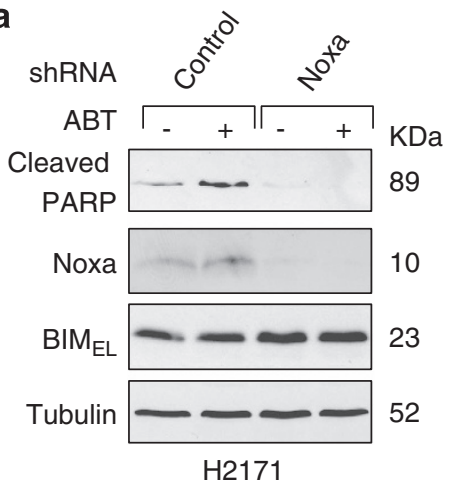

C

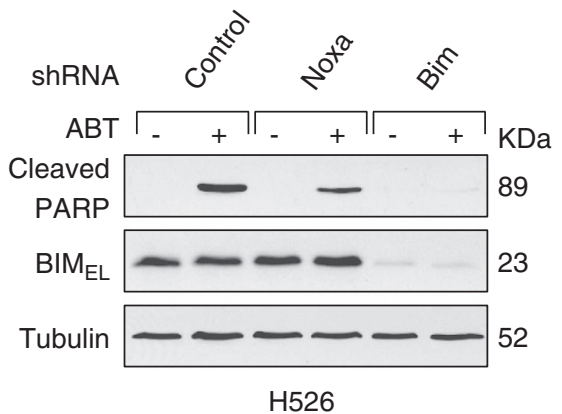

b
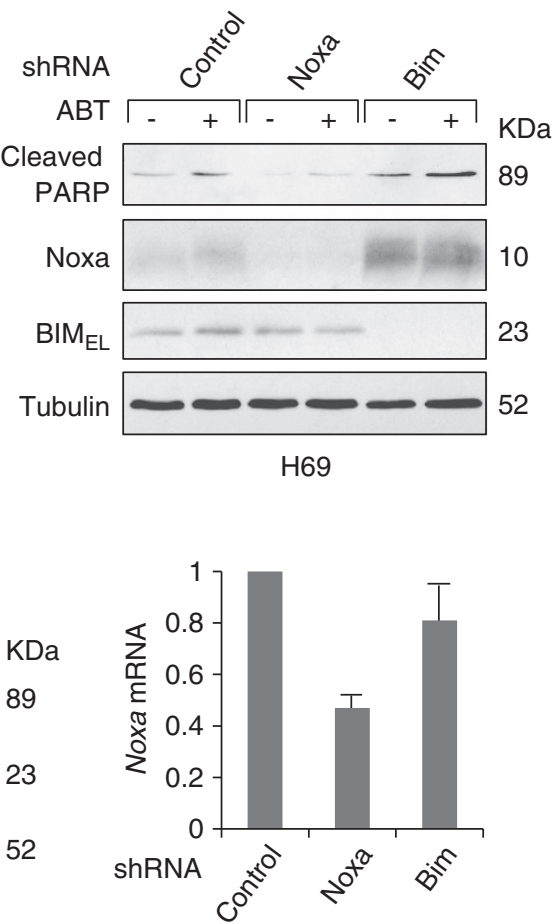

Figure 2 Apoptosis induced by ABT-737 in SCLC cells is regulated by the expression of Noxa and BIM. (a) H2171 cells were infected with lentivirus-encoding shRNA for non-targeting control or Noxa. Cells were then treated with ABT-737 $(100 \mathrm{nM})$ for $24 \mathrm{~h}$, and equal amounts of total extracts were subjected to immunoblot analysis using the indicated antibodies. (b) H69 cells were infected with lentivirus-encoding shRNA for non-targeting control, Noxa, or Bim. Cells were then treated with ABT-737 (100 nM) for $24 \mathrm{~h}$, and equal amounts of total extracts were subjected to immunoblot analysis using the indicated antibodies. (c) H562 cells were infected with lentivirus-encoding shRNA for non-targeting control, Noxa, or Bim. Cells were then treated with ABT-737 $(1 \mu \mathrm{M})$ for $24 \mathrm{~h}$, and equal amounts of total extracts were subjected to immunoblot analysis using the indicated antibodies. The levels of Noxa mRNA before ABT-737 treatment were determined by qPCR (right panel)

anti-Myc antibodies (Figure 5). Exogenous myc-tagged MCL-1 was stably expressed with similar amounts as the endogenous MCL-1 in HeLa cells (Figure 5a). We then introduced Noxa-wt or Noxa-3E into the HeLa cells containing myctagged MCL-1 and fractionated into cytosol and HM fractions. Hela cells expressed undetectable levels of endogenous Noxa, and both Noxa-wt and Noxa-3E were predominantly localized in the mitochondria (Figure $5 \mathrm{~b}$ ). In contrast, both myc-tagged and endogenous MCL-1 were localized less in the cytosol and more at the mitochondria when Noxa-wt was expressed. However, the expression of Noxa-3E did not affect MCL-1 localization compared with HeLa cells without Noxa expression (Figure 5b). The Noxa-dependent MCL-1 translocation was also confirmed by immunofluorescence. Myc-tagged MCL-1 was diffusely distributed in the HeLa cells without the introduction of Noxa-wt (Figure 5c, upper panels). In contrast, myc-tagged MCL-1 had a punctate distribution at the mitochondria when Noxa-wt was expressed (Figure 5c, lower panels). These results indicate that the Noxa-mediated MCL-1 translocation is a common mechanism, which is regulated by the Noxa $\mathrm{BH} 3$ domain.

Noxa induces MCL-1 ubiquitination only when they form a complex at the mitochondria. The protein stability of both Noxa and MCL-1 is known to be regulated by ubiquitination-dependent proteasomal degradation. The phosphorylation status of MCL-1 determines ubiquitination of MCL-1. ${ }^{23,24}$ Thus, we examined whether MCL-1 ubiquitination was regulated by Noxa-wt or mutant expression. Expression of Noxa-wt and Noxa-3E resulted in a series of slower-migrating bands spaced at $\sim 7-\mathrm{kD}$ intervals on a Noxa immunoblot (Figure 6a). In contrast, Noxa-KR, in which all putative lysines for ubiquitination were substituted to arginines, did not show similar bands, suggesting that slower-migrating bands are ubiquitinated Noxa. Slowermigrating bands were also undetectable for Noxa-5A, which is predominantly cytosolic (Figure 6a). We also determined the ubiquitination status of $\mathrm{MCL}-1$ by immunoprecipitation with anti-MCL-1 followed by immunoblot analysis with antiubiquitin antibodies. MCL-1 ubiquitination was strongly enhanced in $\mathrm{H} 209$ cells with Noxa wt but not with mutants disrupting the $\mathrm{BH} 3$ or MTD domains or potential ubiquitination sites (Figure 6b). In cells treated with the proteasome inhibitor MG132, ubiquitinated Noxa and MCL-1 were stabilized, resulting in increased quantities of Noxa-wt, Noxa-3E, and MCL-1 in H209 cells expressing Noxa-wt (Figure 6a). To directly assess the effects of Noxa on MCL1 stability, we monitored the decay of MCL-1 after inhibition of new protein synthesis by cycloheximide treatment. MCL-1 was degraded faster in the presence of Noxa-wt expression than in the absence of Noxa (half-lives, $\sim 0.5$ and $\sim 1.5 \mathrm{~h}$, respectively; Figures $6 \mathrm{c}$ and d). Noxa-dependent modulation of MCL-1 stability was also confirmed in the H2171 SCLC cell line. Downregulation of Noxa by shRNA stabilized MCL-1 (half-lives: control, $\sim 2.5 \mathrm{~h}$; shNoxa, $\sim 4.5 \mathrm{~h}$; Figures $6 \mathrm{e}$ and f). Taken together, these data indicate that Noxa regulates 

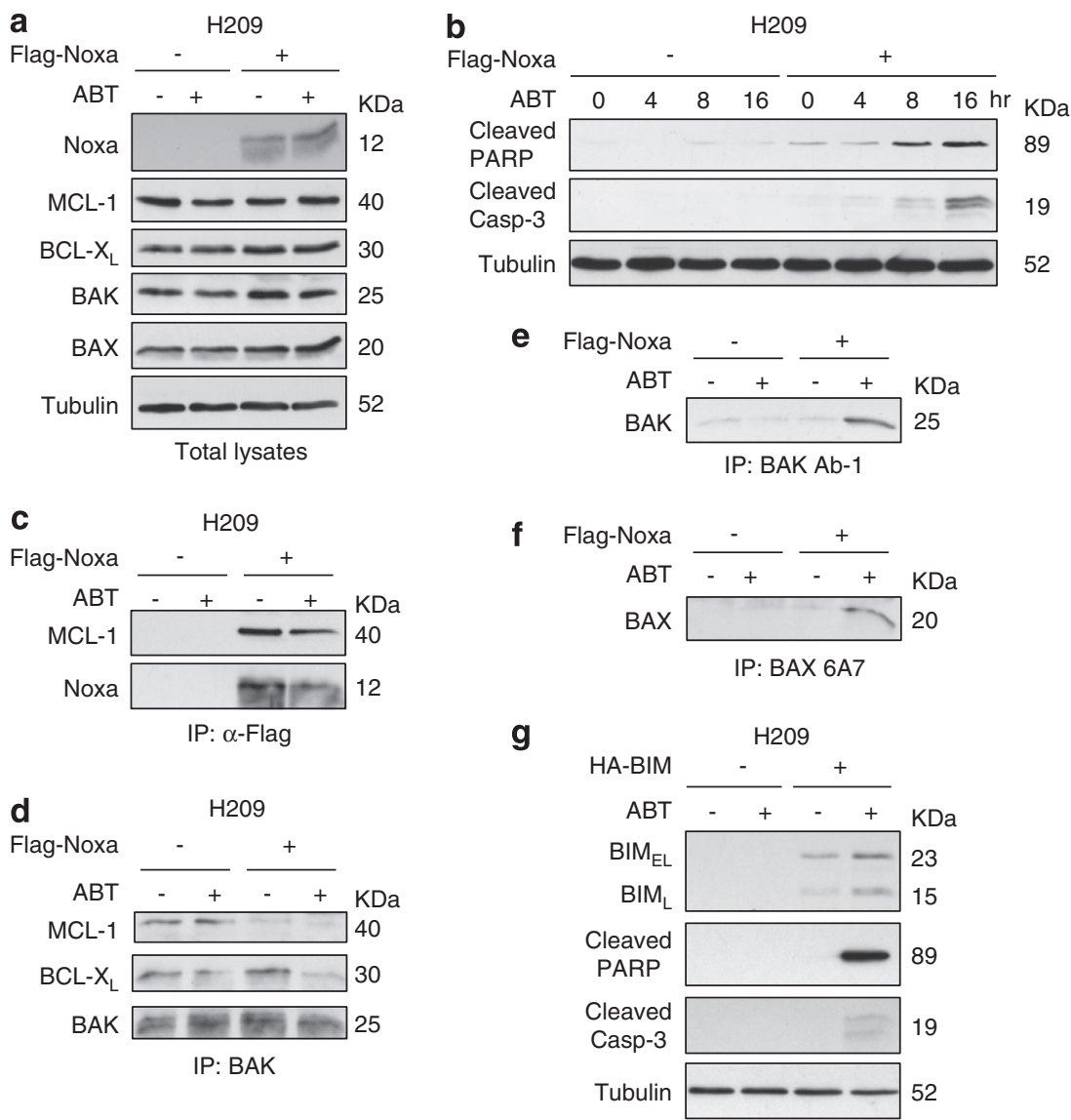

Figure 3 Expression of Noxa or BIM in H209 cells restores the sensitivity to ABT-737. (a) H209 cells were infected with lentivirus-encoding Flag-tagged Noxa cDNA or vector alone as control. Cells were then treated with ABT-737(10 nM) for $16 \mathrm{~h}$. Equal amounts of total extracts were subjected to immunoblot analysis using the indicated antibodies. (b) H209 cells expressing control or Flag-tagged Noxa were treated with ABT-737 (10 nM) for the indicated times, and equal amounts of total extracts were subjected to immunoblot analysis using the indicated antibodies. (c-f) Total cell lysates in (a) were subjected to immunoprecipitation (IP) with (c) anti-Flag, (d) anti-BAK, (e) conformational change-specific anti-BAK (Ab-1), or (f) conformational change-specific anti-BAX (6A7) antibodies. Immunoblot analyses were carried out on precipitated samples with the indicated antibodies. (g) H209 cells expressing control or HA-tagged BIM were treated with ABT-737(10 nM) for $16 \mathrm{~h}$. Equal amounts of total extracts were subjected to immunoblot analysis using the indicated antibodies

MCL-1 stability in a complex process involving modulation of its subcellular localization, phosphorylation, and ubiquitination status.

\section{Discussion}

SCLC is the only solid tumor against which ABT-737 and its orally available derivative ABT-263 are effective as a single agent, albeit with a broad range of sensitivities. ${ }^{10,13,14}$ Although these drugs could eventually result in a significant advance in the treatment of SCLC, considerable work must be done to optimize the likelihood of therapeutic success. The reasons for variable effectiveness must be understood, biomarkers for predicting efficacy must be developed, and the problem of overlapping toxicity with chemotherapy must be overcome. It has been shown that MCL-1, Noxa, and BIM have critical roles in determining $A B T-737$ sensitivity. ${ }^{25-27}$ We first examined the expression of the BCL-2 family proteins in a panel of SCLC cell lines. Among the family proteins, the expression of Noxa and BIM was dramatically different and only Noxa expression correlated with the sensitivity of ABT-737 (Figure 1). Noxa was originally identified as a p53 target gene. ${ }^{28}$ However, p53 expression was undetectable except in $\mathrm{H} 1184$ cells that contain a G334V p53 mutation, ${ }^{29}$ suggesting that Noxa expression in SCLC is p53-independent. Loss of Noxa expression appears to be a tumor-specific event, as the matched BL209, a lymphoblastoid line derived from the same patient as H209, expresses easily detectable levels of Noxa mRNA and protein (Supplementary Figure S4).

We divided SCLC cell lines in four groups based on the levels of Noxa and BIM. Among them, Noxa-high cells were more sensitive to ABT-737, and downregulation of Noxa strongly inhibited apoptosis induced by ABT-737 regardless of the level of BIM expression (Figures $2 a$ and b). In addition, Noxa expression in BIM-null H209 cells restored ABT-737 sensitivity (Figure $3 \mathrm{~b}$ ). Thus, Noxa is a critical molecule determining ABT-737 sensitivity in SCLC cells. In contrast, the significance of BIM for ABT-737 sensitivity depends on the level of Noxa expression. When Noxa expression was high, downregulation of BIM did not affect apoptosis induced by ABT-737 (H69, Figure 2b), suggesting that BIM is dispensable. However, BIM played an important role in ABT-737induced apoptosis when Noxa expression was low 
a

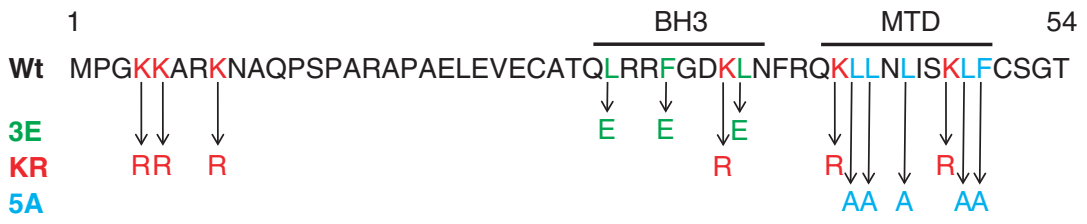

MTD: mitochondrial targeting domain

b

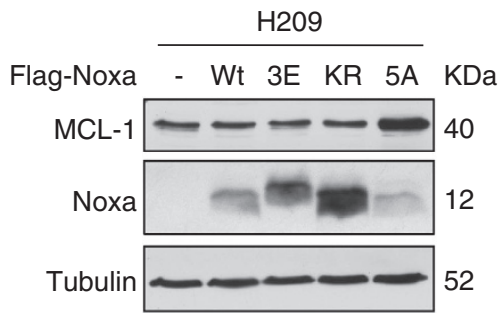

C

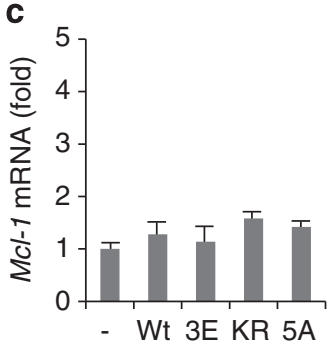

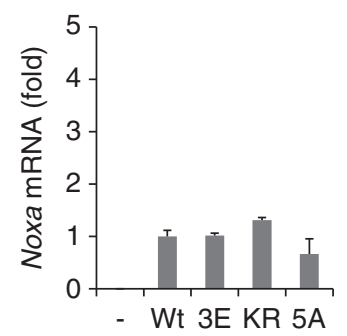

e Flag-Noxa
$\frac{\mathrm{Wt}}{-+} \frac{\mathrm{KR}}{-+\mathrm{KDa}}$

Flag-Noxa - Wt 3E KR 5A KDa

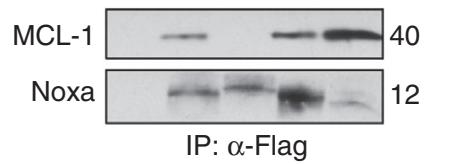

$-++3+$

PARP - - - -

Cleaved

Casp-3

Tubulin $\rightarrow$

$-2$

$\mathrm{H} 209$

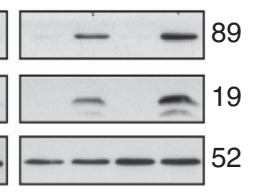
.

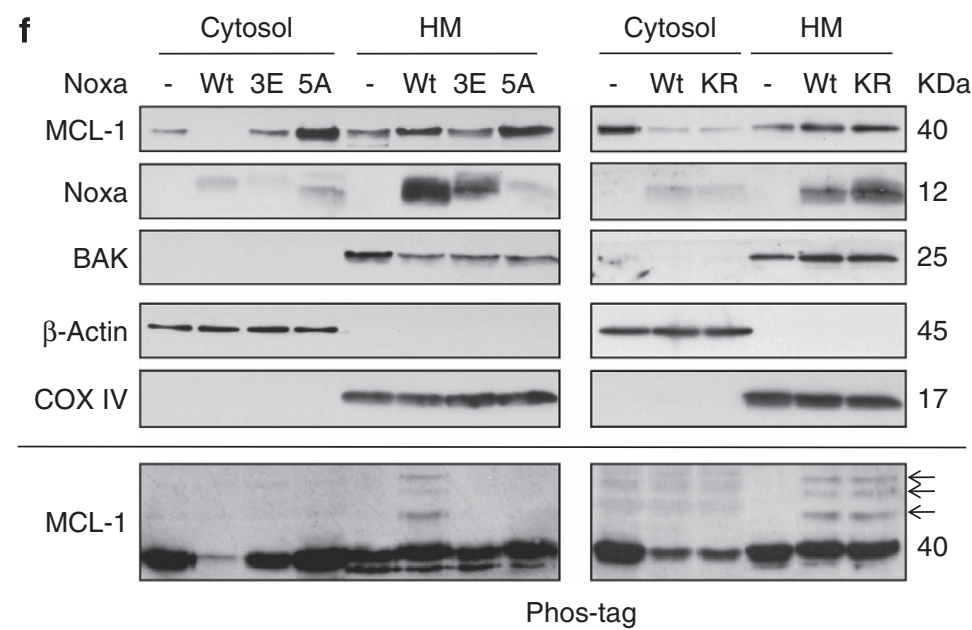

Figure 4 Noxa binds to and recruits MCL-1 from cytosol to mitochondria. (a) Schematic representation of amino-acid sequence of Noxa-wt and the mutants constructed in this study. (b) H209 cells expressing Flag-tagged Noxa-wt or mutants were analyzed by immunoblot using the indicated antibodies. (c) The levels of Mcl-1 or Noxa mRNA in the cells in panel (b) were determined by qPCR. Average values from triplicate samples are shown as representative of two independent experiments. (d) The interaction between Noxa and MCL-1 was examined by co-immunoprecipitation. Total cell lysates in (a) were subjected to immunoprecipitation (IP) with anti-Flag antibodies, and immunoblot analyses were carried out on precipitated samples with the indicated antibodies. (e) H209 cells expressing Noxa-wt or mutants were treated with ABT-737 (10 nM) for $16 \mathrm{~h}$, and equal amounts of total extracts were subjected to immunoblot analysis using the indicated antibodies. (f) H209 cells expressing Noxa-wt or mutants were fractionated into cytosol and $\mathrm{HM}$ fractions. Each fraction was subjected to immunoblot analysis using the indicated antibodies. The integrity of fractionation was determined by $\beta$-actin for cytosol and cytochrome $c$ oxidase IV (COX IV) for HM. Phosphorylated MCL-1 was detected on 10\% SDS-PAGE (sodium dodecyl sulfate-polyacrylamide gel electrophoresis) with $25 \mu \mathrm{M}$ Phos-tag acrylamide (lower panel). Arrows indicate the phosphorylated MCL-1

(H526, Figure 2c). Moreover, the expression of BIM in Noxanull $\mathrm{H} 209$ cells restored ABT-737 sensitivity (Figure $3 \mathrm{~g}$ ). We speculate that the affinity between Noxa and $\mathrm{MCL}-1$ might be stronger than that between BIM and MCL-1. Thus, MCL-1 could be occupied by Noxa in Noxa-high cells and the sensitivity to ABT-737 in these cells becomes Noxa-dependent. On the other hand, most MCL-1 could be bound to BIM when Noxa expression was low and ABT-737 sensitivity could be regulated by both Noxa and BIM.

It has been proposed that Noxa regulates MCL-1 expression. ${ }^{30}$ The introduction of Noxa-wt in H209 cells restored ABT-737 sensitivity by binding to MCL-1 (Figure 3). Noxa-3E, a $\mathrm{BH} 3$ domain mutant, lost the ability to bind to MCL-1 and to induce apoptosis by ABT-737 (Figures $4 d$ and e). Noxa-3E as 
a

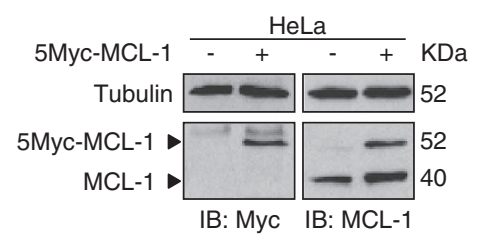

b

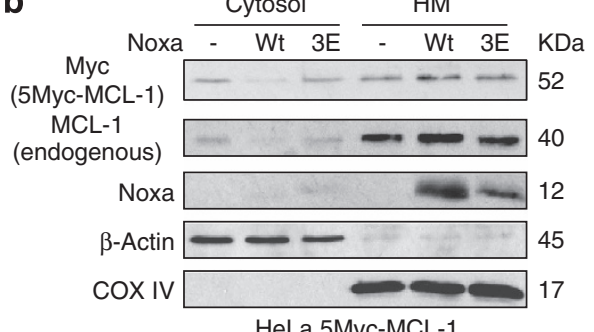

HeLa 5Myc-MCL-1

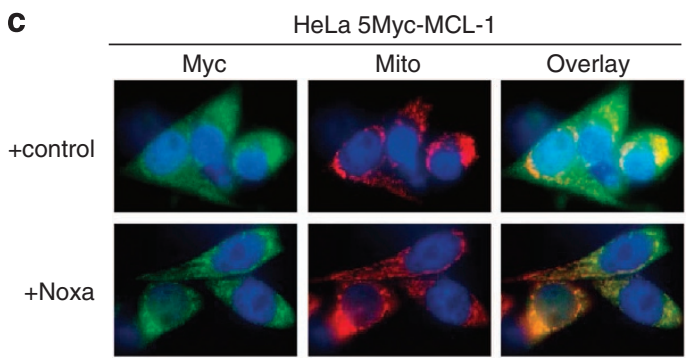

Figure 5 Noxa recruits MCL-1 from cytosol to mitochondria in HeLa cells. (a) HeLa cells expressing Myc-tagged MCL-1 (5Myc-MCL-1) or vector alone as control were analyzed by immunoblot using the indicated antibodies. Arrowheads indicate the positions of Myc-tagged or endogenous MCL-1, respectively. (b) HeLa cells expressing Myctagged MCL-1 (5Myc-MCL-1) were infected with lentivirus encoding Noxa-wt, Noxa-3E, or vector alone as control. Twenty-four hours after infection, cells were fractionated into cytosol and HM fractions. Each fraction was subjected to immunoblot analysis using the indicated antibodies. The integrity of fractionation was determined by $\beta$-actin for cytosol and cytochrome $c$ oxidase IV (COX IV) for HM. (c) HeLa cells expressing 5Myc-MCL-1 were infected with lentivirus encoding Noxa-wt or vector alone as control. Twenty-four hours after infection, Myc-tagged Mcl-1 was immunostained by an anti-Myc (9E10) antibody and mitochondria (Mito) were visualized with Mitotracker Red

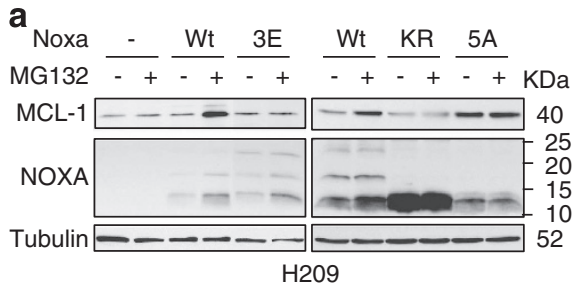

b

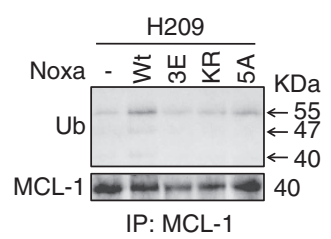

e

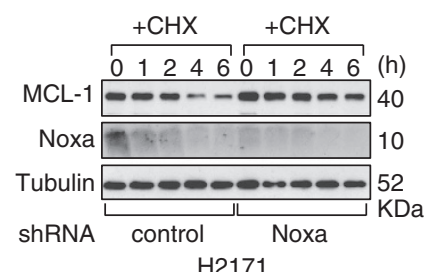

C

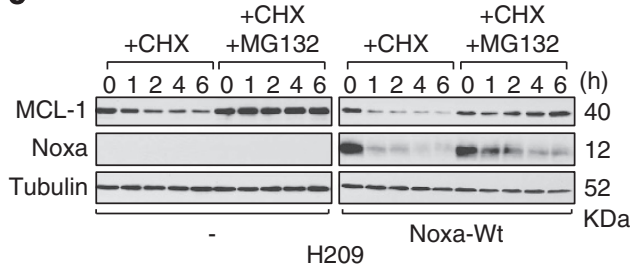

d

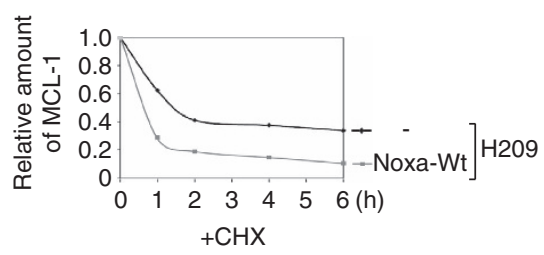

f

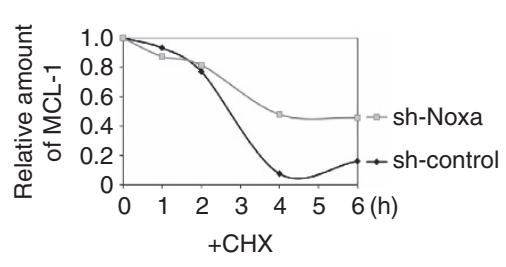

Figure 6 The expression of Noxa-wt enhances Mcl-1 ubiquitination and degradation. (a) H209 cells expressing Noxa-wt or mutants were treated with MG-132 (10 $\mu$ M) for $4 \mathrm{~h}$. Equal amounts of total extracts were subjected to immunoblot analysis using the indicated antibodies. The left panel is a merged image from the single film exposure. (b) H209 cells expressing Noxa-wt or mutants were treated with MG-132 (10 $\mu \mathrm{M})$ for $4 \mathrm{~h}$. Total cell lysates were subjected to immunoprecipitation with anti-MCL-1 antibodies, and immunoblot analysis was carried out on precipitated samples with anti-ubiquitin antibodies. (c) H209 cells expressing control vector ( - ) or Noxa-wt were treated with cycloheximide (CHX, $10 \mu \mathrm{g} / \mathrm{ml})$ in the presence or absence of MG-132 $(10 \mu \mathrm{M})$ for the indicated times. Equal amounts of total extracts were subjected to immunoblot analysis using the indicated antibodies. (d) The relative amount of MCL-1 with CHX treatment in panel (c) was determined by densitometric analysis using the NIH Image J program and was normalized against the amount of tubulin. (e) H2171 cells were infected with lentivirus-encoding shRNA for non-targeting control or Noxa. Cells were treated with $\mathrm{CHX}$ $(10 \mu \mathrm{g} / \mathrm{ml})$ for the indicated times, and equal amounts of total extracts were subjected to immunoblot analysis using the indicated antibodies. (f) The relative amount of MCL-1 in panel (e) was determined by densitometric analysis using the NIH Image J program and was normalized against the amount of tubulin 

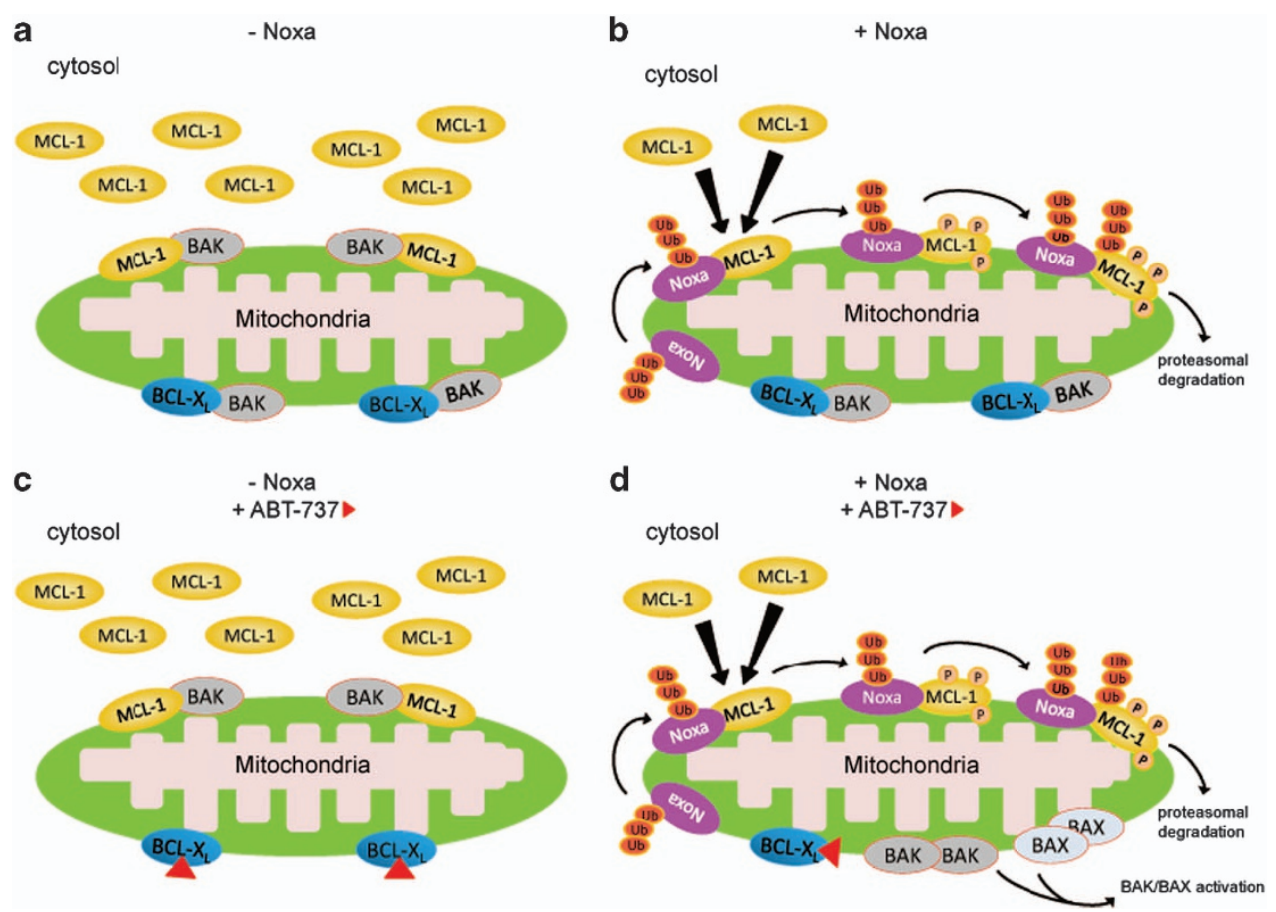

Figure 7 The regulation of MCL-1 by Noxa expression and the mechanisms of ABT-737 sensitivity in H209 cells. See text for details

well as Noxa-wt predominantly localized at the mitochondria. Although the localization of MCL-1 was clearly shifted from the cytosol to the mitochondria when Noxa-wt was expressed, the expression of Noxa-3E did not change MCL-1 localization, compared with $\mathrm{H} 209$ without Noxa (Figure 4f). These data suggest that Noxa recruits MCL-1 through binding with its $\mathrm{BH} 3$ domain. Noxa-mediated MCL-1 translocation was also observed in HeLa cells (Figure 5), indicating a common mechanism. We further examined Noxa and MCL-1 localization using Noxa-5A in which all hydrophobic amino acids in MTD were substituted to alanines. Surprisingly, we found that the level of Noxa-5A protein was lower than that of Noxa-wt, and the level of MCL-1 protein was significantly higher in the cells expressing Noxa-5A (Figure 4b), although the levels of Noxa and MCL-1 mRNA were similar (Figure 4c). Noxa-5A still retained the ability to bind to MCL-1 and enhance ABT737 sensitivity, albeit not to the same degree as Noxa-wt, likely due to lower levels of Noxa-5A and higher levels of MCL-1 (Figures $4 \mathrm{~d}$ and e). MCL-1 predominantly localized in the cytosol with Noxa-5A expression. These results suggest that proper localization of Noxa regulates not only the localization of MCL- 1 by the binding through the Noxa $\mathrm{BH} 3$ domain but also the stability of each protein.

Both Noxa and MCL-1 are labile proteins; the stability is regulated, at least in part, by ubiquitin-dependent proteasome degradation. The expression level of Noxa-KR (a ubiquitination sites mutant) was higher than Noxa-wt, although the levels of Noxa mRNA were similar (Figure 4b), suggesting that Noxa-KR was more stable. Noxa-KR induced more apoptosis with ABT737 than Noxa-wt, as this stable Noxa mutant was still capable of binding to MCL-1 (Figures 4d and e). The localization of Noxa-KR was predominantly at the mitochondria, thus MCL-1 was also translocated to the mitochondria, as similarly observed with Noxa-wt expression (Figure 4f). Interestingly, when Noxa-wt or Noxa-KR was expressed in H209 cells, the MCL-1 phosphorylation at the mitochondria was clearly enhanced (Figure 4f, lower panel). These results suggest that the phosphorylation of MCL-1 occurs at the mitochondria only when it co-localizes with Noxa. Noxa-wt and Noxa-3E, but not Noxa-KR, showed slower-migrating bands on SDS-PAGE, suggesting that slower-migrating bands are ubiquitinated Noxa (Figure $4 \mathrm{f}$ ). Slower-migrating bands were undetectable in Noxa-5A, which predominantly localized in the cytosol. Consistently, when cells were treated with the proteasome inhibitor MG132, the levels of Noxa-wt and Noxa-3E increased, but not those of Noxa-KR and Noxa-5A, supporting the ubiquitination of Noxa-wt and Noxa-3E. These results suggest that Noxa can be ubiquitinated only at the mitochondria, independent of the binding to MCL-1. The ubiquitination of MCL-1 was enhanced only when Noxa-wt was expressed (Figures $6 a$ and $b$ ). These data suggest that for MCL-1 ubiquitination to take place Noxa must localize at the mitochondria, bind MCL-1, and be ubiquitinated. Consistent with its ubiquitination status, the half-life of MCL-1 shortened when Noxa-wt was expressed in H209 cells (Figures $6 \mathrm{c}$ and d). Conversely, the half-life of MCL-1 lengthened when Noxa was downregulated in $\mathrm{H} 2171$ cells (Figures $6 \mathrm{e}$ and f). Of note, MCL-1 became stable with MG132 treatment while Noxa was still degraded, implicating the presence of a ubiquitinindependent pathway for Noxa degradation (Figure 6c). ${ }^{31}$

Based on the above data, we propose the following model for the regulation of Noxa/MCL-1 localization and ubiquitindependent protein stability which, in turn, regulates ABT-737 sensitivity (Figure 7): (a) In cells without Noxa expression, MCL-1 predominantly localizes in the cytosol. The cytosollocalized MCL-1 is neither phosphorylated nor ubiquitinated, 
thus is relatively stable; (b) When Noxa is expressed, this protein is localized at the mitochondria via its mitochondrialtargeting domain. Noxa subsequently becomes ubiquitinated on lysine residues independent of binding to MCL-1. MCL-1 is recruited from the cytosol to the mitochondria by binding to the BH3 domain of Noxa. MCL-1 can then be phosphorylated and ubiquitinated, and as a consequence, it becomes less stable; (c) ABT-737 interacts with BCL- $X_{L}$ and $B C L-2$, resulting in the release of BAK. However, BAK is captured by MCL-1, thus cannot be activated; and (d) When Noxa is expressed, ABT737 binds to BCL- $X_{L}$ and BCL-2 and Noxa binds to MCL-1, resulting in the release of $B A K$ and subsequent activation of BAK and BAX. We speculate that kinase(s) and E3 ligase(s) responsible for Noxa/MCL-1 phosphorylation and ubiquitination, respectively, may co-localize at the mitochondria. These kinase(s) and E3 ligase(s) could be critical modulatory components of Noxa/MCL-1-regulated apoptosis. Further studies of these complex regulatory mechanisms could reveal alternative strategies to enhance the therapeutic index of $\mathrm{BH} 3$ mimetic drugs and overcome chemotherapy resistance in SCLC.

\section{Materials and Methods}

Cell lines and cell culture. Human SCLC cell lines, HeLa, and 293T cells were purchased from the American Type Culture Collection (Manassas, VA, USA).

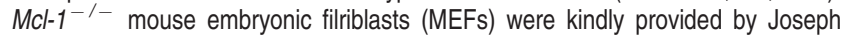
Opferman (St. Jude Children's Research Hospital, Memphis, TN, USA). H69, H128, H526, H82, and H209 were cultured in RPMI 1640 (Life Technologies, Grand Island, NY, USA) supplemented with 10\% heat-inactivated fetal bovine serum (FBS) and $100 \mu \mathrm{g} / \mathrm{ml}$ penicillin G/streptomycin at $37^{\circ} \mathrm{C}$ in a humidified, $5 \% \mathrm{CO}_{2}$ incubator. $\mathrm{H} 1184, \mathrm{H} 2171$, and $\mathrm{H} 2141$ were cultured in DMEM/F12 (Life Technologies) supplemented with $5 \%$ FBS, extra $2 \mathrm{mM}$ L-glutamine, insulin-transferrin-sodium selenite supplement (Roche, Indianapolis, IN, USA) and $100 \mu \mathrm{g} / \mathrm{ml}$ penicillin $\mathrm{G} /$ streptomycin. HeLa, 293T, and $\mathrm{Mcl}-1^{-/-} \mathrm{MEFs}$ were cultured in DMEM supplemented with $10 \%$ FBS and $100 \mu \mathrm{g} / \mathrm{ml}$ penicillin G/streptomycin.

Plasmid construction and virus infection. The lentiviral shRNAexpressing constructs were purchased from Open Biosystems (Huntsville, AL, USA). Noxa cDNAs were cloned into pCDH-EF1-MCS-IRES-neo (System Biosciences, Mountain View, CA, USA). Noxa mutant cDNAs were constructed with QuikChange Lightning Site-Directed Mutagenesis kit (Agilent Technologies, Santa Clara, CA, USA) according to the manufacturer's protocol. The constructs were transfected into 293T packaging cells along with the packaging plasmids, and the lentivirus-containing supernatants were used to transduce SCLC cells. Human Mcl-1 CDNA was synthesized from HeLa cells by RT-PCR. The N-terminal $5 \times$ Myc-tagged Human MCL-1 (5Myc-MCL-1) was constructed by cloning the above Mcl-1 CDNA into pBabe-Hygro encoding a $5 \times$ Myc epitope tag. The constructs were transfected into 293T packaging cells along with the packaging plasmids, and the retrovirus-containing supernatants were used to transduce HeLa cells.

Chemicals and antibodies. Cycloheximide and MG132 were purchased from Millipore (Billerica, MA, USA). Antibodies purchased were as follows: BIM (C34C5), BCL- $\mathrm{X}_{\mathrm{L}}(54 \mathrm{H} 6)$, Cleaved PARP (D64E10) and Cleaved Caspase-3 (5A1E) from Cell Signaling Technology (Danvers, MA, USA); Noxa (114C307.1) from Thermo Fisher Scientific (Waltham, MA, USA); BAX (6A7) from Sigma (St. Louis, MO, USA); MCL-1 from Enzo Life Sciences (Farmingdale, NY, USA); BAX (N-20) and alpha-Tubulin (sc-8035) from Santa Cruz Biotechnology (Santa Cruz, CA, USA); BAK (06-536) and BAK (Ab-1) from Millipore; and Ubiquitin (VU-1) from Life Sensors (Malvern, PA, USA).

Immunoprecipitation and immunoblot analyses. Whole-cell lysates were prepared with CHAPS lysis buffer (20 mM Tris (pH 7.4), $137 \mathrm{mM} \mathrm{NaCl}, 1 \mathrm{mM}$ dithiothreitol (DTT), 1\% CHAPS (3-[(3-cholamidopropyl) dimethylammonio]-1propanesulfonate), a protease inhibitor cocktail, and phosphatase inhibitor cocktails (Sigma)). For immunoprecipitation, equal amounts of protein were precleared with protein A/G beads (Pierce, Rockford, IL, USA) and incubated with the appropriate antibodies on ice for $2 \mathrm{~h}$. Then the antibody complexes were captured with protein $A / G$ beads at $4^{\circ} \mathrm{C}$ for $1 \mathrm{~h}$. After washing three times with the same lysis buffer, the beads were re-suspended in the sample buffer and separated by SDS-PAGE. For western blotting analyses, equal amounts of proteins were loaded on a SDS acrylamide gel, transferred to a nitrocellulose membrane, and analyzed by immunoblotting. Phosphorylated MCL-1 was detected on 10\% SDS-PAGE with $25 \mathrm{mM}$ Phos-tag acrylamide (Wako, Richmond, VA, USA) according to the manufacturer's protocol. ${ }^{22}$

Immunofluorescence. Cells were plated on sterile coverslips and allowed to attach for $24 \mathrm{~h}$. Cells were then serum starved for $48 \mathrm{~h}$, washed, fixed with cold methanol for $15 \mathrm{~min}$, washed with PBS for $5 \mathrm{~min}$, and blocked in $5 \%$ bovine serum albumin, $0.1 \%$ (v/v) tween20 in PBS for $30 \mathrm{~min}$ at ambient temperature. Cells were incubated with monoclonal anti-Myc (9E10) antibody (Santa Cruz Biotechnology) diluted $1: 200$ in blocking buffer overnight at $4{ }^{\circ} \mathrm{C}$, washed three times with PBS, and incubated with a FITC-conjugated anti-mouse antibody (1:5000 dilution) for $1 \mathrm{~h}$ at ambient temperature. Coverslips were mounted using Vectashield (Vector Labs, Burlingame, CA, USA) and viewed with a Zeiss Axiovert 200 microscope (Thornwood, NY, USA) with excitation at $488 \mathrm{~nm}$. Mitochondria were labeled with $100 \mathrm{nM}$ MitoTracker Red CMXRos (Cell Signaling Technology) for $30 \mathrm{~min}$.

Subcellular fractionation. The cells were resusupended in hypotonic buffer A (200 mM mannitol, $70 \mathrm{mM}$ sucrose, $10 \mathrm{mM}$ Hepes (pH 7.5), 1 mM EGTA, and $1 \times$ protease inhibitor cocktail) on ice for $15 \mathrm{~min}$. The cells were destroyed by passing through a 25-gauge needle 15 times, and then centrifuged at $1000 \times g$ for $10 \mathrm{~min}$ at $4^{\circ} \mathrm{C}$ to eliminate unbroken cells and nuclei. The supernatant was centrifuged at $10000 \times g$ for $30 \mathrm{~min}$ at $4^{\circ} \mathrm{C}$. The pellet was lysed in RIPA buffer as the $\mathrm{HM}$ fraction. The supernatant was further centrifuged at $100000 \times g$ for $1 \mathrm{~h}$ at $4{ }^{\circ} \mathrm{C}$. The supernatant was saved as the soluble cytosol (S100) fraction.

Cell viability assay. SCLC cells were seeded in triplicate in microtiter plates (96 wells) with a concentration of $4 \times 10^{4}$ cells per well in $100-\mu$ medium and were treated with $0-10 \mu \mathrm{M} \mathrm{ABT}-737$ for $72 \mathrm{~h}$. Cell survival was measured by the WST-1 assay, which is based on the tetrazolium salt WST-1 (4-[3-(4-iodophenyl)2-(4-nitrophenyl)-2H-5-tetrazolio]-1,3-benzene disulphonate), according to the manufacturer's instruction (Roche).

Quantitative RT-PCR. Total RNA was extracted with the RNeasy kit (Qiagen, Valencia, CA, USA) from SCLC cell lines. One-microgram of RNA was reverse-transcribed by High Capacity cDNA Reverse Transcription Kit (Applied Biosystems, Carlsbad, CA, USA) according to the manufacturer's instructions. Using Taqman Gene Expression Assay probe/primer (Hs00197982 for Bim, $\mathrm{Hs} 00560402$ for Noxa, and Hs03043899 for Mcl-1 (Applied Biosystems)), cDNAs were amplified in a fluorescence thermocycler (Applied Biosystems; 7500HT Fast Real-time PCR system) and were analyzed based on the expression level of GADPH with SDS2.2 software (Applied Biosystems).

Statistical analysis. Values represent the means \pm S.D. for three separate experiments. The significance of differences between experimental variables was determined using the Student's $t$-test. Values were considered statistically significant at $P<0.05$.

\section{Conflict of Interest}

The authors declare no conflict of interest.

Acknowledgements. We thank Joseph Opferman for providing us $\mathrm{MCl}^{-1^{-/-}}$ MEFs and the members of Steven Grant's lab for valuable comments. This work was supported by the Massey Cancer Center Pilot Project grant (to GWK and $\mathrm{HH}$ ) and in part by NIH R01CA134473 (to HH). WN was partly supported by Nippon Medical School.

\footnotetext{
1. Jackman DM, Johnson BE. Small-cell lung cancer. Lancet 2005; 366: 1385-1396.

2. Aisner J. Extensive-disease small-cell lung cancer: the thrill of victory; the agony of defeat. J Clin Oncol 1996; 14: 658-665.
} 
3. Johnson DH, Carbone DP. Increased dose-intensity in small-cell lung cancer: a failed strategy? J Clin Oncol 1999; 17: 2297-2299.

4. Glisson BS, Kurie JM, Perez-Soler R, Fox NJ, Murphy WK, Fossella FV et al. Cisplatin, etoposide, and paclitaxel in the treatment of patients with extensive small-cell lung carcinoma. J Clin Oncol 1999; 17: 2309-2315.

5. Kelly K, Lovato L, Bunn Jr PA, Livingston RB, Zangmeister J, Taylor SA et al. Cisplatin, etoposide, and paclitaxel with granulocyte colony-stimulating factor in untreated patients with extensive-stage small cell lung cancer: a phase II trial of the Southwest Oncology Group. Clin Cancer Res 2001; 7: 2325-2329.

6. Youle RJ, Strasser A. The BCL-2 protein family: opposing activities that mediate cell death. Nat Rev Mol Cell Biol 2008; 9: 47-59.

7. Chipuk JE, Moldoveanu T, Llambi F, Parsons MJ, Green DR. The BCL-2 family reunion. Mol Cell 2010; 37: 299-310.

8. Ben-Ezra JM, Kornstein MJ, Grimes MM, Krystal G. Small cell carcinomas of the lung express the Bcl-2 protein. Am J Pathol 1994; 145: 1036-1040.

9. Ikegaki N, Katsumata M, Minna J, Tsujimoto Y. Expression of bcl-2 in small cell lung carcinoma cells. Cancer Res 1994; 54: 6-8.

10. Oltersdorf T, Elmore SW, Shoemaker AR, Armstrong RC, Augeri DJ, Belli BA et al. An inhibitor of $\mathrm{Bcl}-2$ family proteins induces regression of solid tumours. Nature 2005; 435 : $677-681$.

11. Tahir SK, Yang X, Anderson MG, Morgan-Lappe SE, Sarthy AV, Chen J et al. Influence of Bcl-2 family members on the cellular response of small-cell lung cancer cell lines to ABT-737. Cancer Res 2007; 67: 1176-1183.

12. Tse C, Shoemaker AR, Adickes J, Anderson MG, Chen J, Jin S et al. ABT-263: a potent and orally bioavailable Bcl-2 family inhibitor. Cancer Res 2008; 68: 3421-3428.

13. Shoemaker AR, Mitten MJ, Adickes J, Ackler S, Refici M, Ferguson D et al. Activity of the Bcl-2 family inhibitor ABT-263 in a panel of small cell lung cancer xenograft models. Clin Cancer Res 2008; 14: 3268-3277.

14. Gandhi L, Camidge DR, Ribeiro de Oliveira M, Bonomi P, Gandara D, Khaira D et al. Phase I study of Navitoclax (ABT-263), a novel Bcl-2 family inhibitor, in patients with small-cell lung cancer and other solid tumors. J Clin Oncol 2011; 29: 909-916.

15. Deng J, Carlson N, Takeyama K, Dal Cin P, Shipp M, Letai A. BH3 profiling identifies three distinct classes of apoptotic blocks to predict response to ABT-737 and conventional chemotherapeutic agents. Cancer Cell 2007; 12: 171-185.

16. Lin X, Morgan-Lappe S, Huang X, Li L, Zakula DM, Vernetti LA et al. 'Seed' analysis of off-target siRNAs reveals an essential role of Mcl-1 in resistance to the small-molecule Bcl-2/Bcl-XL inhibitor ABT-737. Oncogene 2007; 26: 3972-3979.

17. Hauck P, Chao BH, Litz J, Krystal GW. Alterations in the Noxa/Mcl-1 axis determine sensitivity of small cell lung cancer to the BH3 mimetic ABT-737. Mol Cancer Ther 2009; 8: 883-892.
18. Willis SN, Chen L, Dewson G, Wei A, Naik E, Fletcher Jl et al. Proapoptotic Bak is sequestered by $\mathrm{Mcl}-1$ and $\mathrm{Bcl}-\mathrm{xL}$, but not Bcl-2, until displaced by BH3-only proteins. Genes Dev 2005; 19: 1294-1305.

19. Chen L, Willis SN, Wei A, Smith BJ, Fletcher JI, Hinds MG et al. Differential targeting of prosurvival $\mathrm{Bcl}-2$ proteins by their $\mathrm{BH} 3$-only ligands allows complementary apoptotic function. Mol Cell 2005; 17: 393-403.

20. Baou M, Kohlhaas SL, Butterworth M, Vogler M, Dinsdale D, Walewska R et al. Role of NOXA and its ubiquitination in proteasome inhibitor-induced apoptosis in chronic lymphocytic leukemia cells. Haematologica 2010; 95: 1510-1518.

21. Seo YW, Shin JN, Ko KH, Cha JH, Park JY, Lee BR et al. The molecular mechanism of Noxa-induced mitochondrial dysfunction in p53-mediated cell death. J Biol Chem 2003; 278: 48292-48299.

22. Kinoshita E, Kinoshita-Kikuta E, Takiyama K, Koike T. Phosphate-binding tag, a new tool to visualize phosphorylated proteins. Mol Cell Proteomics 2006; 5: 749-757.

23. Thomas LW, Lam C, Edwards SW. Mcl-1; the molecular regulation of protein function. FEBS Lett 2010; 584: 2981-2989.

24. Inuzuka H, Fukushima H, Shaik S, Liu P, Lau AW, Wei W. Mcl-1 ubiquitination and destruction. Oncotarget 2011; 2: 239-244.

25. Dai $Y$, Grant $\mathrm{S}$. Targeting multiple arms of the apoptotic regulatory machinery. Cancer Res 2007; 67: 2908-2911.

26. Kuroda J, Taniwaki M. Involvement of BH3-only proteins in hematologic malignancies. Crit Rev Oncol Hematol 2009; 71: 89-101.

27. Davids MS, Letai A. Targeting the B-cell lymphoma/leukemia 2 family in cancer. J Clin Oncol 2012; 30: 3127-3135.

28. Oda E, Ohki R, Murasawa H, Nemoto J, Shibue T, Yamashita T et al. Noxa, a BH3-only member of the Bcl-2 family and candidate mediator of p53-induced apoptosis. Science 2000; 288: 1053-1058.

29. Phelps RM, Johnson BE, Inde DC, Gazdar AF, Carbone DP, McClintock PR et al. NClNavy Medical Oncology Branch cell line data base. J Cell Biochem Suppl 1996; 24: 32-91.

30. Adams JM, Cory S. The Bcl-2 apoptotic switch in cancer development and therapy. Oncogene 2007; 26: 1324-1337.

31. Craxton A, Butterworth M, Harper N, Fairall L, Schwabe J, Ciechanover A et al. NOXA, a sensor of proteasome integrity, is degraded by $26 \mathrm{~S}$ proteasomes by an ubiquitinindependent pathway that is blocked by MCL-1. Cell Death Differ 2012; 19: 1424-1434.

(c) (i) $\odot$ Cell Death and Disease is an open-access journal published by Nature Publishing Group. This work is licensed under a Creative Commons Attribution-NonCommercialNoDerivs 3.0 Unported License. To view a copy of this license, visit http://creativecommons.org/licenses/by-nc-nd/3.0/

Supplementary Information accompanies this paper on Cell Death and Disease website (http://www.nature.com/cddis) 